\title{
Some new results in porous silicon
}

\author{
V K JAIN, AMITA GUPTA, C R JALWANIA, ADARSH KUMAR, \\ G K SINGHAL, O P ARORA, D S AHUJA, P P PURI, R SINGH and \\ M PAL \\ Solid State Physics Laboratory, Lucknow Road, Delhi 110054, India
}

\begin{abstract}
Efforts have been made to see the effect of some standard microelectronic processing steps on porous silicon. Our diffusion experiments for making $p-n$ junctions confirm that this material can withstand high temperatures of the order of $800^{\circ} \mathrm{C}$ to $1000^{\circ} \mathrm{C}$. A new technique for photolithography has been suggested to obtain porous silicon in selected areas. Etch stop method to control the thickness of the porous layer and an organic protective layer for porous silicon have also been suggested. Models proposed by other workers to explain luminescence in porous silicon are not sufficient to explain many experimental observations. A hybrid model is suggested.
\end{abstract}

Keywords. Porous silicon; $\mathrm{p}-\mathrm{n}$ junction.

\section{Introduction}

The recent observation of visible photoluminescence from porous silicon has invoked wide interest in this material. Currently it is one of the most intensively investigated materials due to its potential as a building block in optoelectronics. In spite of wide-ranging investigations of its properties, a consistent explanation for the various observations has not yet been established, including the basic mechanisms of light emission from silicon nanostructures. In order to develop the technology for making light-emitting devices several attempts have been made to fabricate electroluminescent (EL) structures but these have generally given feeble light. We report a strong EL from a $\mathrm{p}-\mathrm{n}$ junction formed by diffusion in porous silicon. This paper reports some new results in this direction and also a technique for photolithography in porous silicon. Efforts have also been made to explain some of the experimental observations.

\section{Formation of porous silicon}

The porosity in silicon is commonly obtained by partial anodic dissolution of silicon in hydrofluoric acid (HF - ethanol) solution. Several attempts (Smith and Collins 1992; Jung et al 1993) have been made to explain the chemistry of porosity generation in silicon, but the mechanisms involved in pore size formation are not fully understood. It is also not clear why the walls between the pores are so remarkably resistant to further chemical attack of the electrolyte.

In our experiments (Jain et al 1993) silicon wafers having aluminium deposited on the back side by vacuum evaporation were covered with acid-resistant wax and then anodized in $48 \% \mathrm{HF}$ - ethanol (1:1) solution. Platinum was used as the cathode. The anodization current was kept between 10 and $15 \mathrm{~mA} / \mathrm{cm}^{2}$. Currents higher than $50 \mathrm{~mA} / \mathrm{cm}^{2}$ result in electropolishing of silicon. Good porosity is obtained on p-type silicon in dark or light but on n-type silicon it is obtained only in the presence of intense light indicating that holes play an important role in pore formation. 
The thickness of the porous layer is determined by the anodization current and time. However, better control can be obtained by using an etch stop technique which is useful for making thin porous layers. Here silicon wafer with a $\mathrm{p}-\mathrm{n}$ junction instead of a plain silicon wafer is used. The thickness of the porous layer can be controlled up to the junction depth. First, an n-p junction is made in silicon by diffusion, and after proper cleaning the porosity is generated by the same process as that explained above. The silicon sample was made anode and the contact was taken from the back (Al-coated) side of the wafer. The experimental arrangement is shown in figure 1 . The front $n$-side of the sample formed a Schottky junction with HF. The barrier height between n-side and HF is high, due to reverse bias, which does not permit the flow of anodization current. In presence of light the Schottky barrier height decreases and the anodization starts, giving porosity on n-type $\mathrm{Si}$. The important point is that the

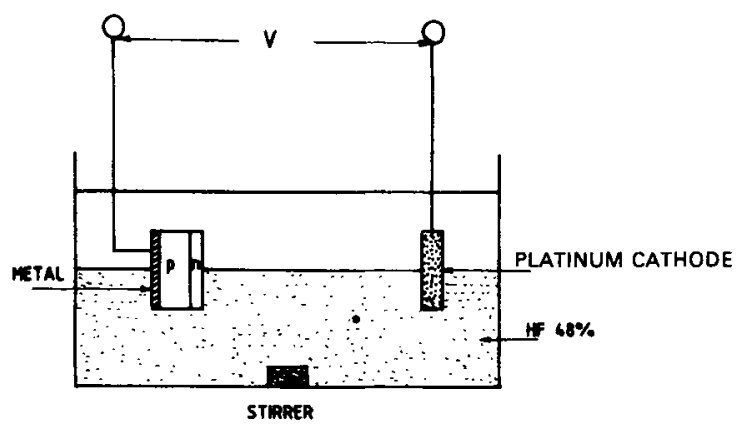

Figure 1. Experimental arrangement for etch stop technique.

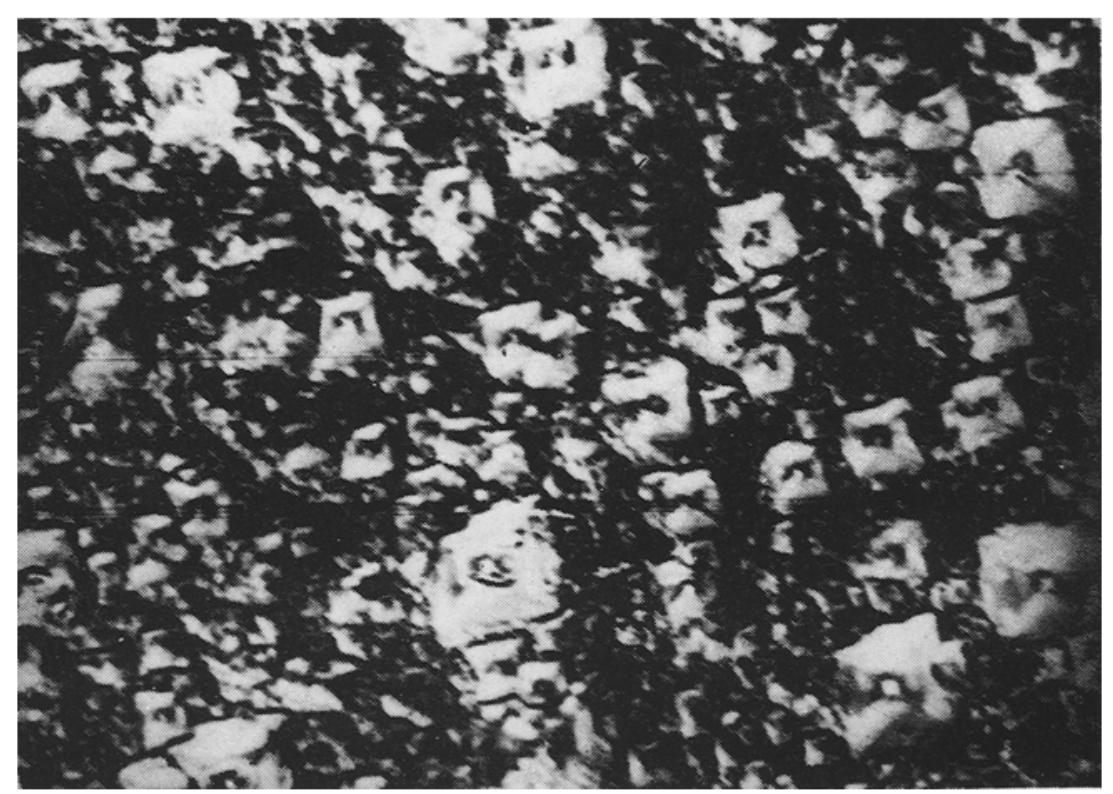

Figure 2. SEM photograph of texturized porous silicon $(M=1000)$. 
positive voltage applied to $\mathrm{p}$-side is kept such that the HF - p Si Schottky junction does not become forward biased. This restricts the porosity formation only up to the diffused region. Porous layers of different thicknesses have been made by this technique.

The porosity has also been generated on texturized silicon wafer. The pores are generated in the walls of the pyramids of the texturized surface resulting in increased PL intensity. The SEM picture in figure 2 shows the formation of pores.

\subsection{Formation of $p-n$ junction in porous silicon}

Chen and Bosman (1993) and Steiner et al (1993) made $\mathrm{p}-\mathrm{n}$ junction in porous silicon by ion implantation in the silicon wafer and then generated the porosity by the usual technique. Jain et al (1993) proposed a new technique and made $\mathrm{p}^{+}-\mathrm{n}$ and $\mathrm{n}^{+}-\mathrm{p}$ junctions by diffusion of counterdopants in porous silicon at high temperatures. The diffusion of phosphorus in p-type from a $\mathrm{POCl}_{3}$ source and boron in $\mathrm{n}$-type porous silicon from a boron nitride solid source is carried out at $850^{\circ} \mathrm{C}$ and $975^{\circ} \mathrm{C}$ respectively in a continuous flow of nitrogen. This formed $n^{+}-p$ and $p^{+}-n$ structures. The high low junctions $\mathrm{p}^{+}-\mathrm{p}$ and $\mathrm{n}^{+}-\mathrm{n}$ have also been made.

\subsection{Photolithography in porous silicon}

Peng et al (1993) studied the effect of standard microelectronic processing steps, such as thermal and chemical oxidation, ion implantation and reactive ion etching, on porous silicon, before making attempts to integrate these porous silicon devices with silicon integrated chips. They also found that after oxidation the PL shows blue shift and is more stable. Low-dose ion implantation does not change PL but after annealing the intensity gets reduced. Our diffusion experiments for making $\mathrm{p}-\mathrm{n}$ junctions also confirm that this material can withstand high temperatures of the order of $800^{\circ} \mathrm{C}$ to $1000^{\circ} \mathrm{C}$ except that it shows some reduction in PL intensity.

One of the most important steps in device fabrication is the photolithography (PLG) process and masking to get small geometries having good porosity. In general, in photolithography masking is achieved by silicon dioxide, silicon nitride, photoresists or metal layers. But in the present case, these materials cannot stand in HF for the long period required for porosity generation. A new technique has been developed which we name waxolithography.

Figure 3 shows the flowchart for this process. In this method first a layer of acid-resistant wax was deposited on silicon wafer by spin coating. After properly drying it, a layer of photoresist was spin-coated on this wax layer. Mask aligner was used to expose the circular window patterned mask in photoresist and a special developer, which does not affect the wax layer, was used to generate the pattern in photoresist layer. By using another suitable developer which does not affect the photoresist mask, windows were opened in wax layer. On completion of this process photoresist was removed and porosity was generated in silicon only through these wax windows by the same technique described earlier. In this case also the back of the wafer was coated with aluminium and protected with acid-resistant wax. Dot pattern of $800 \mu \mathrm{m}$ diameter and 'H' shape pattern of $200 \mu \mathrm{m}$ have been generated. The dots are shown in figure 4 glowing under UV light. 

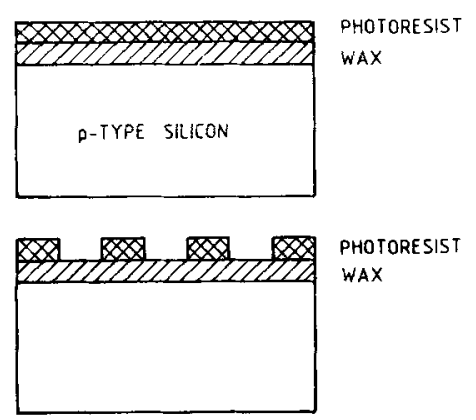

PHOTORESIST

WAX
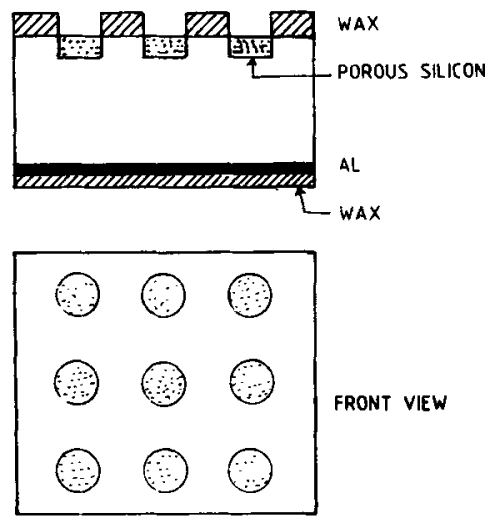

Figure 3. Waxolithography for generating fine geometries in porous silicon.

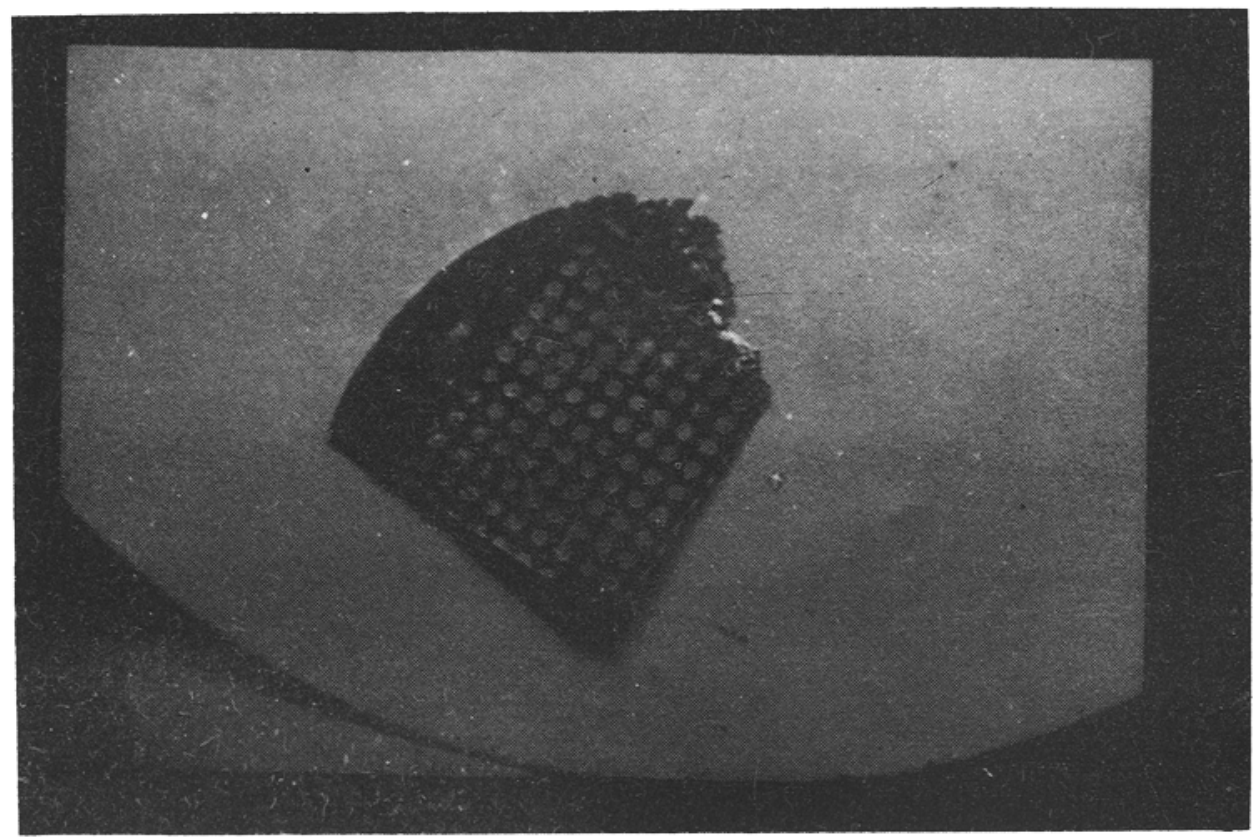

Figure 4. Porous dots formed by waxolithography glowing red under UV lamp 


\subsection{Protective layer for porous silicon}

Porous layer formed on the surface of the silicon wafer is highly fragile and brittle. It is very important to protect the layer after device formation from atmospheric degradation and to keep it stable for a long time. Some efforts have been made by different workers in this field to embed this material in $\mathrm{SiO}_{2}$ matrix. But growing of silicon dioxide by thermal oxidation or by sputtering damages the porous material. We have developed a very stable protective layer of organic compounds which can be deposited on porous silicon by a simple spin-on technique. These layers have been optimized for the required thickness to give minimum reduction in PL intensity.

Measurements of photoluminescence show that even after a year there is no degradation in intensity and no peak shift in these porous silicon wafers having protective layer. The organic layer is also stable. Detailed measurements will be published elsewhere.

\section{Photoluminescence}

Visible photoluminescence is the most important and commonly observed property of porous silicon. Generally a bright red or orange glow can be seen by placing the sample in ultraviolet light. A typical spectrum as shown in figure 5, is taken by exciting the sample with a $488 \mathrm{~nm}$ argon ion laser. Some workers have found that the peak wavelength can be shifted from near-infrared to green and even to blue by varying the anodization conditions. Both n-type and p-type samples of $2 \mathrm{ohm}-\mathrm{cm}$ resistivity give similar PL spectrum.

The photoluminescence has also been studied on our samples by spectral scanning photoluminescence technique (Krawczyk et al 1992) using $\mathrm{He}-\mathrm{Ne}$ laser to scan the $100 \mu \mathrm{m} \times 100 \mu \mathrm{m}$ area of porous silicon made on high-resistivity silicon ( $5000 \mathrm{ohm}-\mathrm{cm}$, n-type) wafer. It gives a complete picture of integrated photoluminescence mapping. The PL mapping was done at different areas of the sample at room temperature and

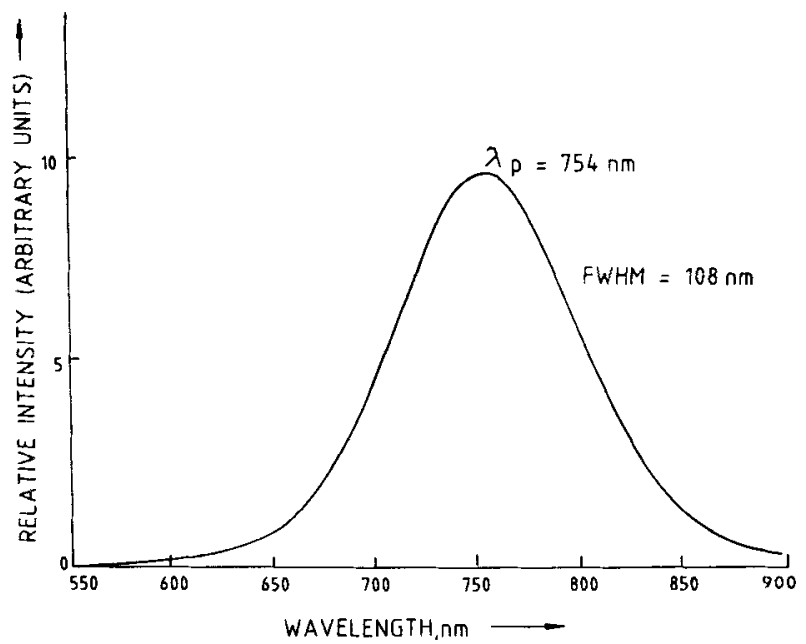

Figure 5. Typical photoluminescence spectrum from porous silicon. 


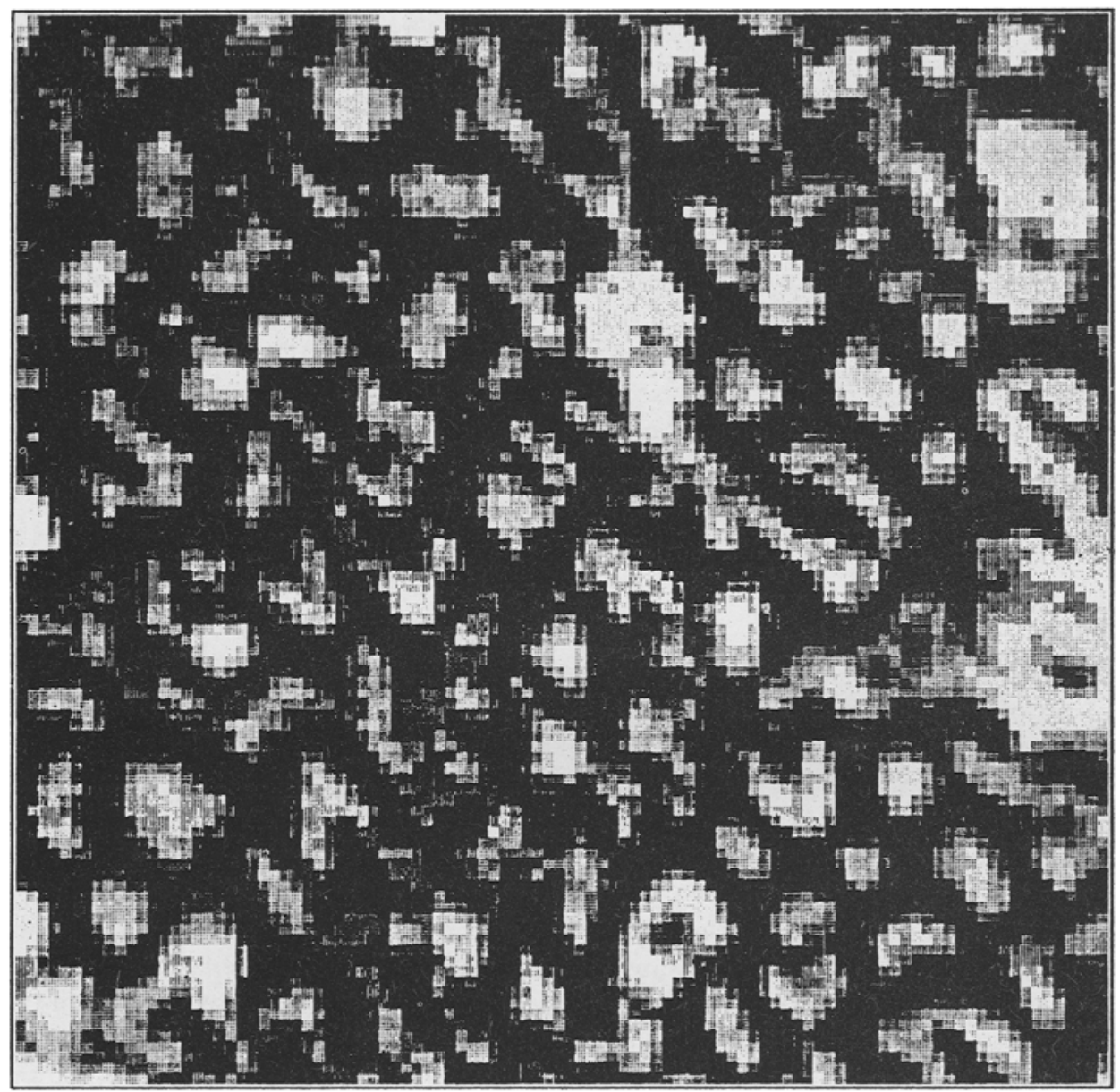

Figure 6. Photoluminescence scanning map.

a typical picture is shown in figure 6 . The dark black areas show intense photoemission while shaded areas have less, and completely unshaded areas do not give any emission.

In recent work (Tsai 1991), it has been shown that the PL intensity of porous silicon decreases sharply on annealing at a temperature of $300^{\circ} \mathrm{C}$. FTIR measurements also show that this is the temperature at which hydrogen gets desorbed from the silicon hydride species. Jain et al $(1993,1994)$ reported the first observation of photoluminescence from porous silicon after counterdoping by diffusion at high temperatures $\left(850-1000^{\circ} \mathrm{C}\right)$. In these investigations it was found that $\mathrm{p}^{+}-n$ and $\mathrm{n}^{+}-\mathrm{p}$ junctions formed in PS show photoluminescence but $n^{4}-n$ and $p^{+}-p$ give no PL.

\subsection{Resistivity and photoconductuity in porous silicon}

The major problem which restricts the use of this material in making any optoelectronic device is its high resistivity and consequently practical difficulty in 


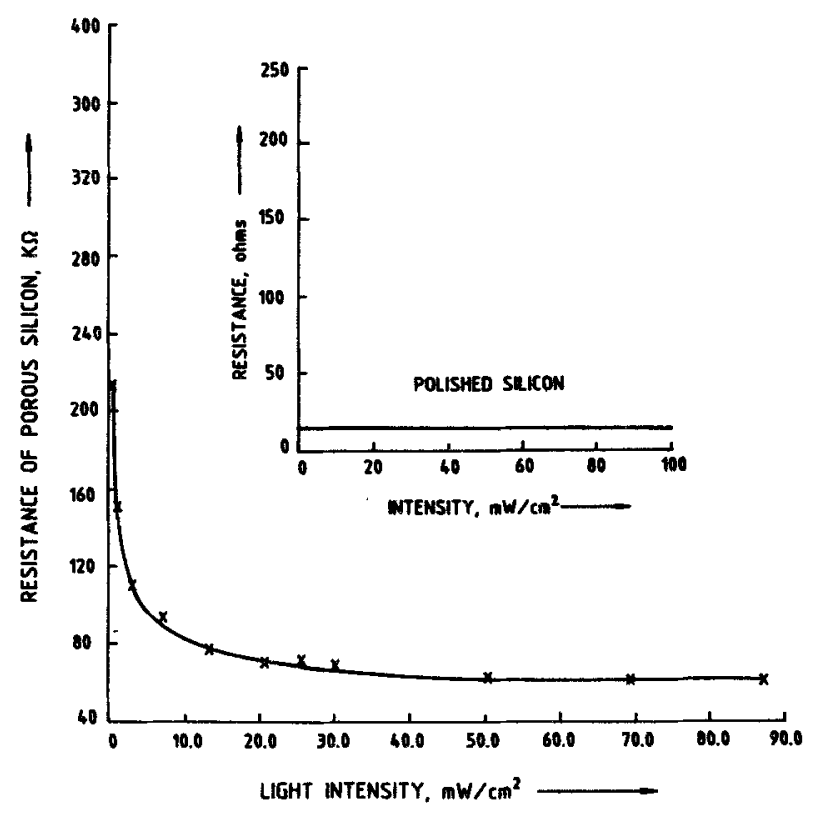

Figure 7. Variation of resistance with light intensity.

making good ohmic contacts. Diffusion of impurities has given a major breakthrough in reducing the resistance of porous layer.

Electrical measurements as shown in figure 7 indicate very high resistance of these porous layers which agrees with the results reported by other workers in this field. The inset shows that plain silicon wafer has about $15 \mathrm{ohm}$ resistance between two metallic ohmic contacts at a distance of $2 \mathrm{~mm}$. Generation of porosity has increased the resistance to about 220 kilo-ohms in dark between the two contacts at the same distance. Photoconductivity measurements as shown in figure 7 indicate that the resistance of plain silicon does not change with increase of light intensity. However, porous silicon resistance drops from $220 \mathrm{kiloohm}$ to $60 \mathrm{kiloohm}$.

The resistance drops to a very low value after diffusion of phosphorus in p-type and boron in $n$-type porous silicon. Diffusion was also performed in plain silicon wafer under the same conditions to compare the photoconducting behaviour with diffused porous silicon. The resistivity is low and the behaviour is the same as in the case of diffused plain silicon sample. It was confirmed that the top metal contacts made for the resistance measurements do not penetrate through the porous layer and the contact resistance is low.

\subsection{Electroluminescence in porous silicon-light-emitting diodes}

Observation of photoluminescence in silicon is exciting, but the development of optoelectronic devices requires that the material should respond to electrical signals. Therefore, considerable efforts worldwide are aimed at obtaining electroluminescence from this material.

Richter et al (1991) and Koshida and Koyama (1992) studied the electroluminescence by making the metal Schottky contact with porous silicon layer. Richter et al (1991) 
found an EL peak at $680 \mathrm{~nm}$ when a current of $5 \mathrm{~mA}$ was passed through the sample at $200 \mathrm{~V}$. Koshida and Koyama (1992) found rectifying behaviour in this junction and EL peak at $680 \mathrm{~nm}$. Namavar et al (1992) observed electroluminescence in ITO-PS Schottky-like structures, but these devices give feeble and unstable electroluminescence. Recently in 1993, Chen and Bosman (1993) observed visible-light emission from porous silicon homojunction. They made an $n^{+}-p^{+}$junction by ion implantation in porous silicon wafer. The porosity was generated after the junction was formed by ion implantation. The EL was seen through a $100-\AA$-thick semi transparent gold layer.

In the same year Steiner et al (1993) also made a diode with PS following the same technique of ion implantation in wafer and then generating the porosity in the junction. The light emission has been seen through thin metal pads and on application of low voltage. At higher voltages $(25 \mathrm{~V})$ red-orange glow was seen with naked eye under room illumination but within minutes the series resistance increased and also caused a decline in light intensity. Lee and Peng (1993) formed blue-light-emittir diodes in porous silicon. The emission has again been observed through a semitransparent gold electrode.

Here we report a stable bright red $E L$ in $n^{+}-p$ and $\mathrm{p}^{+}-\mathbf{n}$ junctions made by diffusion in porous silicon on application of $5 \mathrm{~V}$ and $300-400 \mathrm{~mA}$ current. The important observation is the brightness and stability of the electroluminescence. The voltage was applied across the junction in forward direction. The exact power requirement will be predicted only after optimizing many factors like area of porous silicon, contact resistance and contact materials, etc. Presently efforts are in the direction of reducing the total power consumption. It was also found that the sample gives continuous bright electroluminescence for an indefinite period when it is kept at liquid nitrogen temperature. It was also observed that high low junctions, i.e. $p^{+} p$ and $n^{+} n$ in porous silicon do not give any electroluminescence.

In order to study the electrical properties of this junction, $5 \mathrm{~mm}$ dia $\mathrm{Ti} / \mathrm{Pd} / \mathrm{Ag}$ contacts were evaporated through a metal mask on top of the diffused $n^{+}$porous

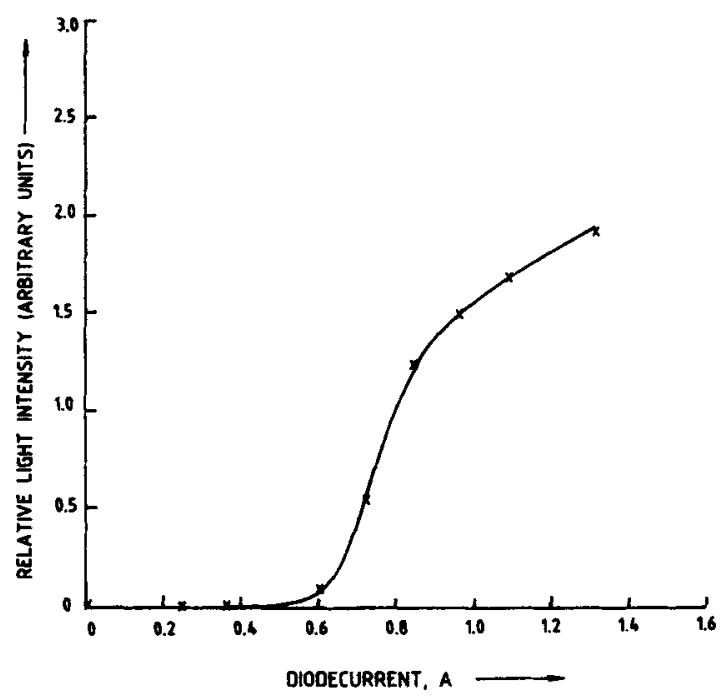

Figure 8. Variation of emitted light intensity as a function of applied current. 
silicon layer and $\mathrm{Al}$ at the back of the wafer. The variation of the emitted light intensity as a function of diode current is shown in figure 8.

\section{Theory}

Since the discovery of porous silicon, the most interesting aspect is the strong light emission at room temperature. Several models have been proposed by various authors, which have been reviewed recently (Searson et al 1992; Jung et al 1993).

One group believes that photoluminescence has a chemical origin resulting from the formation of molecules on the large surface area of PS. One of the suggested candidates is the hydrogenated amorphous phase (Abeles and Tiedje 1983; Wolford et al 1983) of silicon. There is evidence that silicon hydride complexes can give visible photoluminescence at room temperature and the optical band gap of the binary $\mathrm{Si}: \mathrm{H}$ materials increases almost linearly with increasing hydrogen content. The same concept can be extended to porous silicon. Another group (Brandt et al 1992) favours siloxene $\left(\mathrm{Si}_{6} \mathrm{O}_{3} \mathrm{H}_{6}\right)$ and its derivatives. Six-fold silicon rings in siloxene structure play the key role in their luminescence property. Considerable information exists about siloxene and its derivatives which are known to have outstanding luminescence efficiency. The colour of light could be tuned by changing the molecular structure by varying fabrication conditions. The electronic structures of these compounds have been calculated and optical properties predicted, including PL and Raman spectra which fit well with those of PS. The reduction in PL intensity with evolution of hydrogen at about $400^{\circ} \mathrm{C}$ is also consistent with these chemical models.

However, these models fail to explain the presence of PL after treatment at higher temperatures during oxidation and diffusion to make $\mathrm{p}-\mathrm{n}$ junctions. These compounds are expected to decompose at such temperatures. These models alsc cannot explain the occurrence when samples prepared such that they have no hydrogen (Petrova-Koch et al 1992) and no oxygen (Tischler and Collins 1992) also show strong PL. This seems to suggest that oxygen and hydrogen are not the essential constituents in PS for luminescence in the visible range.

One of the most widely suggested ideas is based on the concept of quantum confinement. Porous silicon structures consist of silicon wires, and as the porosity is increased the thickness of the wires decreases to nanometre sizes. TEM results show that wafers of porous silicon having $80 \%$ porosity have average wire width between 30 to $40 \AA$. Read et al (1992) in their calculations for optical properties of porous silicon found that by reducing the size of the silicon wires, the conduction band minima are upshifted in energy by a large amount, because of their small mass in the confinement plane. The band gap of these wires is expected to be direct and sufficient to give the observed visible photoluminescence. Support to this model also comes from the observed blue shift of PL peak from longer etching duration or after oxidation.

Though the quantum size model explains some of the properties of PS, it fails to explain many other observations. No direct relationship between particle size present in PS and visible PL has yet been established. Samples having $20 \%$ porosity also show visible PL. The quantum size model also cannot explain the reduction in PL upon heating up to $400^{\circ} \mathrm{C}$ and also again appearance of PL after oxidation or diffusion at higher temperatures. Changes in PL spectra on selected chemical treatment also cannot be explained easily by this model. 
We suggest a hybrid model to phenomenologically explain the properties of porous silicon based on the model proposed by Koch (1993). It is assumed that porous silicon has a spongy-type structure consisting of quantum size wires. The surface of these wires is passivated with hydrogen which reduces surface recombination. By changing the diameter one can change the colour of emission. The defect states which originate PL in PS could be surface states on the enormous area of PS. On oxidation at high temperatures, PL shifts from red to blue colour. These results suggest that there are two types of centres responsible for PL in porous silicon: red centres due to surface states which give PL near $700 \mathrm{~nm}$ while blue centres due to core of the silicon wire which give blue response. In this three-level luminescent state model, there are two excited states, one representing a nanocrystalline core state (quantum confinement) and the other a surface state. In this structure UV is absorbed in the silicon core due to the enhancement of the band gap and photocarriers generated in the silicon core transfer to the surface state. The carriers recombine radiatively giving visible luminescence equivalent to the energy of these surface states. After oxidation blue region is enhanced because carriers generated in silicon core cannot be transferred to the surface states as the band gap of $\mathrm{SiO}_{2}$ of $\approx 8 \mathrm{eV}$ is large.

In case of electroluminescence, Koshida and Koyama (1992) suggested that top metal contact of Au or ITO forms Schottky junction with porous layer, while porous silicon layer- $\mathrm{Si}$ substrate interface acts as a heterojunction of two different semiconductors. When a bias voltage is applied most of the potential drops across the PS layer because of its high resistivity. When the applied voltage is sufficiently high, electrons from the top and holes from the substrate $\mathrm{Si}$ are injected in porous layer by tunnelling effect. These carriers recombine and emit light. After counterdiffusion in porous silicon the Schottky junction between PS and $\mathrm{Si}$ is replaced by a shallow $\mathrm{p}-\mathrm{n}$ junction. The top $\mathrm{n}^{+}$or $\mathrm{p}^{+}$porous silicon layer is highly doped because of diffusion of impurities in silicon wires from all sides of the wire. Direct radiative transition is possible. The flow of carriers becomes easy by diffusion instead of tunnelling and their radiative recombination in thinned silicon wires is possible at low voltage and currents, which results in bright red luminescence.

\section{Conclusion}

A method has been suggested to control the thickness of the porous layer. It was observed by other workers in this field and also by us that heating at temperatures higher than $300^{\circ} \mathrm{C}$ reduces $\mathrm{PL}$ intensity in porous silicon. Diffusion of counterdopant in PS at high temperatures $\left(800-1000^{\circ} \mathrm{C}\right)$ has been carried out which still gives photoluminescence. A new technique for photolithography has been suggested to obtain porous silicon in selective areas. We have also developed an organic protective layer for porous silicon. Many models have been proposed to explain PL and EL in PS but even the most acceptable model based on quantum confinement is not sufficient to explain many experimental observations. A hybrid model is suggested.

\section{Acknowledgement}

The authors are thankful to Dr Vikram Kumar, Director, SSPL, for his valuable guidance. The authors are also thankful to Dr S K Krawczyk, Scantek, France for his 
help in photoluminescence mapping, Shri G D Sharda, SSPL, for SEM measurements and Dr T Srinivasan for the photoluminescence spectra.

\section{References}

Abeles B and Tiedje T 1983 Phys. Rev. Lett. 512003

Brandt M S, Fuchs H D, Stutzmann M, Weber J and Cardona M 1992 Solid State Commun. 81307

Chen Z and Bosman G 1993 Appl. Phys. Lett. 62708

Jain V K et al 1993 Bull. Mater. Sci. 16239

Jain V K, Gupta A, Kumar A, Singhal G K and Kumar V 1994 J. Pure Appl. Opt. 19943225

Jung K H, Shih S and Kwong D L 1993 J. Electrachem, Soc. 1403046

Koch F 1993 Mater Res. Soc. Symp. Proc. 283197

Koshida N and Koyama H 1992 Appl. Phys. Lett. 60347

Krawczyk S K, Krafft F, Klingelh 'O' fer C, Garrigues M and Schohe K 1992 Semi. Cond. Sci. Technol. A7 73-A79

Lee M K and Peng K R 1993 Appl. Phys. Lett. 623159

Namavar F, Maruska H P and Kalkhoran N M 1992 Appl. Phys. Lett. 602514

Peng C, Tsybeskov L and Fauchet P M 1993 Mater. Res. Soc. Symp. Proc. 283121

Petrova-Koch V, Muschik T, Kux A, Meyer B K, Koch F and Lehmann V 1992 Appl. Phys. Lett. 61943

Read A J, Needs R J, Canham L T, Calcott P D J and Qteish A 1992 Phys. Rev. Lett. 691232

Richter A, Steiner P, Kozlewski F and Lang W 1991 Electron Device Lett. 12691

Searson P C, Mckaulay J M and Prokes S M 1992 J. Electrochem. Soc. 1393373

Smith R L and Collins S D 1992 J. Appl. Phys. 71 R1

Steiner P, Kozlowski F and Lang W 1993 Appl. Phys. Lett. 622700

Tischler M A and Collins R T 1992 Solid State Commun. 84819

Tsai C 1991 Appl. Phys. Lett. 592814

Wolford D J, Reimer J A and Scott B A 1983 Appl. Phys. Lett. 42369 